\title{
Ideal Hausdorff Space
}

\author{
C.R.Parvathy ${ }^{1}$ and E.Divya ${ }^{2}$ \\ ${ }^{I}$ Department of Mathematics, P.S.G.R.Krishnammal college for women, Coimbatore-641004. \\ ${ }^{2}$ Department of Mathematics, P.S.G.R.Krishnammal college for women, Coimbatore-641004.
}

\begin{abstract}
The aim of this paper is to study some properties of ideal Hausdorff space. We introduce some new concepts in ideal topological space such as convergence of sequences and the concepts of Hausdorff axiom in ideal topological space.
\end{abstract}

Keywords: Ideal topological space, Hausdorff space, continuous, open set

\section{Introduction}

Ideals in topological spaces have been considered since 1930. In 1990, once again Jankovie and Hamlett, initiated the application of topological ideals in the generalization of most fundamental properties in general topology. The concept of ideals in topological space was first introduced by Kuratowski and Vaidhyanathswamy. Further Hamlett and Jankovic in and studied the properties

of ideal topological space. They have also obtained a new topology from original ideal topological space. Modak and Bandyopadhyay in 2007 have defined generalized open sets.

The contributions of Hamlett and Jankovic in Ideal topological spaces the generalization of some important properties in general topology via topological ideals. The properties like decomposition of continuity, separation axioms, connectedness, compactness and resolvability have been generalized using the concept of ideals in topological spaces.

A ideal topological space $(X, \tau, I)$ means a tpological space $(X, \tau)$ with an ideal I defined on $X$. Let $(X, \tau)$ be a topological space with an ideal I defined on X.

\section{Preliminaries}

Definition 2.1: An ideal I on a nonempty set $X$ is a collection of subset of $X$ which satisfies the following properties:

(i) $\mathrm{A} \in \mathrm{I}$ and $\mathrm{B} \subseteq \mathrm{A}$ implies $\mathrm{B} \in \mathrm{I}$.

(ii) $\mathrm{A} \in \mathrm{I}$ and $\mathrm{B} \subseteq \mathrm{I}$ implies $\mathrm{A} \cup \mathrm{B} \in \mathrm{I}$.

A topological space $(X, \tau)$ with an ideal I on $X$ is called ideal topological space and is denoted by $(X, \tau, I)$. Let $Y$ be a subset of $X$.

Definition 2.2: A ideal topological space $(X, \tau, I)$ is called g-Hausdorff iff for any two distinct points $\mathrm{p}$ and $\mathrm{q}$ of $\mathrm{X}$, there exist disjoint gf-open sets $\mathrm{U}$ and $\mathrm{V}$ of $\mathrm{X}$ such that $\mathrm{p} \in \mathrm{U}$ and $\mathrm{q} \in \mathrm{V}$.

Definition 2.3: Let $(X, \tau, I)$ be a ideal topological space, $\left(x_{n}\right) \subset X$ be a sequence and $x_{0} \in X .\left(x_{n}\right)$ is called converges to $x_{0}$ in $(X, \tau, I)$ if for all $A \in \mathcal{N}\left(x_{0}\right)$ there exists $n_{0} \in \mathbb{N}$ such that $x_{n} \in A$ for all $n \geq n_{0}$.

Definition 2.4: Let $(X, T, I)$ be a ideal topological space. A subcollection $\beta$ of $T$ is called a base for $T$ if every member of $\mathrm{T}$ can be expressed as a union of members of $\beta$.

Definition 2.5: Let $(\mathrm{X}, \mathrm{T}, \mathrm{I})$ be a ideal topological space. A subcollection $\mathcal{S}$ of $\mathrm{T}$ is said to be a subbase for $\mathrm{T}$ if the family of all finite intersections of members of $\mathcal{S}$ forms a base for $\mathrm{T}$.

Definition 2.6: Let $\left(X, T_{1}, I\right)$ and $\left(Y, T_{2}, I\right)$ be two ideal topological spaces. A mapping $f:\left(X, T_{1}, I\right) \rightarrow\left(Y, T_{2}, I\right)$ is called continuous if $\mathrm{f}^{-1}(\mathrm{~A}) \in \mathrm{T}_{1}, \forall A \in \mathrm{T}_{2}$. A mapping $\mathrm{f:}\left(\mathrm{X}, \mathrm{T}_{1}, \mathrm{I}\right) \rightarrow\left(\mathrm{Y}, \mathrm{T}_{2}, \mathrm{I}\right)$ is called open if $\mathrm{f}(\mathrm{A}) \in$ $\mathrm{T}_{2}, \forall \mathrm{A} \in \mathrm{T}_{1}$.

Definition 2.7: A function $\mathrm{f}:(\mathrm{X}, \tau, \mathrm{I}) \rightarrow(\mathrm{Y}, \sigma, \mathrm{I})$ is called closed if the image of each closed set of $\mathrm{X}$ is closed in Y.

Definition 2.8: Let $\left\{\left(\mathrm{X}, \mathrm{T}_{\mathrm{i}}, \mathrm{I}\right)\right\}_{\mathrm{i} \in \mathrm{j}}$ be a family of ideal topological spaces. Then the initial ideal topology on $\mathrm{X}$ $\left(=\prod_{i \in J} X_{i}\right)$ generated by the family $\left\{(p, q)_{i}\right\}_{i \in J}$ is called product ideal topology on $X$. The product ideal topology is denoted by $\prod_{i \in J} T_{i}$. 
Definition 2.9: Let $(X, \tau, I)$ be a ideal topological space. A subset $A$ of $X$ is called $g$-closed if $\operatorname{cl}(A) \subseteq U$ whenever $\mathrm{A} \subseteq \mathrm{U}$ and $\mathrm{U}$ is open in $\mathrm{X}$.

\section{Hausdorff in Ideal Topological Spaces}

Definition 3.1: Let $(X, \tau, I)$ be an ideal topological space and $x, y \in X$ such that $x \neq y$. $(X, \tau, I)$ is called ideal Hausdorff space if there exist open sets $\mathrm{A}$ and $\mathrm{B}$ such that $\mathrm{x} \in \mathrm{A}, \mathrm{y} \in \mathrm{B}$ and $\mathrm{A} \cap \mathrm{B}=\Phi$.

Theorem 3.2: Let $(X, \tau, I)$ be an ideal topological space and $(Y, \sigma)$ be Hausdorff. If $f:(X, \tau, I) \rightarrow(Y, \sigma)$ is injective and continuous, then $(\mathrm{X}, \tau, \mathrm{I})$ is ideal Hausdorff

Proof: Let $x$ and $y$ be any two distinct points of $X$. Then $f(x)$ and $f(y)$ are different points of $Y$ because $f$ is injective. Since $Y$ is Hausdorff, there exist disjoint open sets $U$ and $V$ in $Y$ containing $f(x)$ and $f(y)$ respectively. Since $\mathrm{f}$ is continuous and $\mathrm{U} \cap \mathrm{V}=\phi, \mathrm{f}^{-1}(\mathrm{U})$ and $\mathrm{f}^{-1}(\mathrm{~V})$ are disjoint open sets in $\mathrm{X}$ such that $\mathrm{x} \in \mathrm{f}^{-1}(\mathrm{U})$ and $\mathrm{y} \in \mathrm{f}^{-1}(\mathrm{~V})$. Hence $\mathrm{X}$ is ideal Hausdorff.

Theorem 3.3: If $(X, \tau, I)$ is an ideal Hausdorff space and $f:(X, T, I) \rightarrow\left(Y, T^{*}, I\right)$ is injective, surjective and open then $\left(\mathrm{Y}, \mathrm{T}^{*}, \mathrm{I}\right)$ is ideal Hausdorff space.

Proof: Let $\mathrm{y}_{1}, \mathrm{y}_{2} \in Y$ such that $\mathrm{y}_{1} \neq \mathrm{y}_{2}$. Then $\mathrm{f}\left(\mathrm{y}_{1}\right)$ and $\mathrm{f}\left(\mathrm{y}_{2}\right)$ are different points of $Y$. Since $\mathrm{f}$ is surjective there exist $x_{1}, x_{2} \in X$ such that $f\left(x_{1}\right)=y_{1}, f\left(x_{2}\right)=y_{2}$ and $x_{1} \neq x_{2}$. From hypothesis $(X, T, I)$ is an ideal Hausdorff space, so there exist $A, B \in T$ such that $x_{1} \in A, x_{2} \in B$ and $A \cap B=\Phi$. So for each $f\left(x_{1}\right)=$ $y_{1}, f\left(x_{2}\right)=y_{2}$. This implies that $f\left(x_{1}\right)=y_{1} \in f(A), f\left(x_{2}\right)=y_{2} \in f(B)$. Since $f$ is open, then $f(A), f(B) \in T^{*}$ and $f$ is injective. $f(A) \cap f(B)=f(A \cap B)=\Phi$. Thus $\left(Y, T^{*}, I\right)$ is ideal Hausdorff space.

Theorem 3.4: In ideal Hausdorff space, a sequence converges to a unique point.

Proof: Assuming that $x$ and $y$ are two distinct points then $\left(x_{n}\right)$ converges to and $y$ and $x \neq y$. Since $(X, T, I)$ is an ideal Hausdorff space there exist $A, B \in T$ such that $x \in A, x \in B$ and $A \cap B=\Phi$. Since $x_{n}$ converges to $x$ and $A$ is neighborhood of $x$, then there exist $n_{1} \in \mathbb{N}$ such that $x_{n} \in A$ for all $n \geq n_{1}$. Since $x_{n}$ converges to $y$ and $B$ is neighborhood of $y$, then there exist $n_{2} \in \mathbb{N}$ such that $x_{n} \in B$ for all $n \geq n_{2}$. Let $n_{0}=\max \left(n_{1}, n_{2}\right)$ then for all $\mathrm{n} \geq \mathrm{n}_{0} . \mathrm{x}_{\mathrm{n}} \in \mathrm{A}$ and $\mathrm{x}_{\mathrm{n}} \in \mathrm{B}$. Hence $\mathrm{A} \cap \mathrm{B} \neq \Phi$. This is a contradiction.

Theorem 3.5: Let $(X, \tau, I)$ be a ideal topological space and $(Y, \sigma, I)$ be g-Hausdorff. If $f:(X, \tau, I) \rightarrow(Y, \sigma, I)$ is injective and gc-irresolute, then $(\mathrm{X}, \tau, \mathrm{I})$ is g-Hausdorff.

Proof: Let $x$ and $y$ be any two distinct points of $X$. Then $f(x)$ and $f(y)$ are different points of $X$ because $f$ is injective. Since $Y$ is g-Hausdorff, there exist disjoint g-open sets $U$ and $V$ in $Y$ containing $f(x)$ and $f(y)$ respectively. Since $\mathrm{f}$ is gc-irresoulte and $\mathrm{U} \cap \mathrm{V}=\phi, \mathrm{f}^{-1}(\mathrm{U})$ and $\mathrm{f}^{-1}(\mathrm{~V})$ are disjoint $\mathrm{g}$-open sets in $\mathrm{X}$ such that $\mathrm{x} \in \mathrm{f}^{-1}(\mathrm{U})$ and $\mathrm{y} \in \mathrm{f}^{-1}(\mathrm{~V})$. Hence $\mathrm{X}$ is g-Hausdorff.

Theorem 3.6: $X$ and $Y$ are ideal Hausdorff space then $X \times Y$ is Hausdorff space.

Proof: Let $X$ and $Y$ be ideal Hausdorff spaces. Let $\left(x_{1}, y_{1}\right),\left(x_{2}, y_{2}\right) \in X \times Y$ and $\left(x_{1}, y_{1}\right) \neq\left(x_{2}, y_{2}\right)$. So $x_{1} \neq$ $\mathrm{x}_{2}$ or $\mathrm{y}_{1} \neq \mathrm{y}_{2}$. Assume that $\mathrm{x}_{1} \neq \mathrm{x}_{2}$. Since $X$ is ideal Hausdorff space there exist $A, B \in T$ such that $x_{1} \in A$, $\mathrm{x}_{2} \in \mathrm{B}$ and $\mathrm{A} \cap \mathrm{B}=\Phi$. Then $\mathrm{A} \times \mathrm{C}$ and $\mathrm{B} \times \mathrm{Y}$ are open set on $\mathrm{X} \times \mathrm{Y}$. Hence $\left(\mathrm{x}_{1}, \mathrm{y}_{1}\right) \in \mathrm{A} \times \mathrm{C},\left(\mathrm{x}_{2}, \mathrm{y}_{2}\right) \in \mathrm{B} \times \mathrm{C}$ and $(\mathrm{A} \times \mathrm{C}) \cap(\mathrm{B} \times \mathrm{C})=\Phi$.

Theorem 3.7: Let A be a compact set in ideal Hausdorff space $(X, \tau, I)$ then A is closed.

Proof: Let $x \in A^{c}$. For each $y \in A$. We have $x \neq y$. So there are disjoint open sets $U$ and V. So that $x \in U$ and $y \in V$. Then $\{V: y \in A\}$ is an open cover of A. Let $\left\{v_{1}, v_{2}, \ldots . v_{n}\right\}$ be a finite subcover. Then $\bigcap_{i=1}^{n} U_{i}$ is an open set containing $\mathrm{x}$ and contained in $\mathrm{A}^{\mathrm{c}}$. Thus $\mathrm{A}^{\mathrm{c}}$ is open and $\mathrm{A}$ is closed.

[1]. Banu Pazar Varol, Halis Aygun (2010)

\section{References}

[2]. Clay Shonkwilers, Topology Final, University of Pennsylvania.

[3]. Ernest Michael, Topologies on spaces of subsets, Trans. Amer. Math. Soc. 71(1951), 152-182.

[4]. N.Levine, Generalized closed sets in Topology, Rend.Circ.Math.Palermo (2), (19) (1970), 89-96

[5]. Willard General topology. Addison Wesley(1970). 